\title{
Correlation of $\mathrm{O}(1 s)$ and $\mathrm{Fe}(2 p)$ near edge $\mathrm{x}$-ray absorption fine structure spectra and electrical conductivity of $\mathrm{La}_{1-x} \mathrm{Sr}_{x} \mathrm{Fe}_{0.75} \mathrm{Ni}_{0.25} \mathrm{O}_{3-\delta}$
}

\author{
Selma Erat, ${ }^{1,2, a)}$ Artur Braun, ${ }^{1, a)}$ Alejandro Ovalle, ${ }^{1}$ Cinthia Piamonteze, ${ }^{3}$ Zhi Liu, ${ }^{4}$ \\ Thomas Graule, ${ }^{1,5}$ and Ludwig J. Gauckler ${ }^{2}$ \\ ${ }^{1}$ Laboratory for High Performance Ceramics, EMPA-Swiss Federal Laboratories for Materials Testing \\ and Research, $\mathrm{CH}-8600$ Dübendorf, Switzerland \\ ${ }^{2}$ Department of Materials, Nonmetallic Inorganic Materials, ETH Zurich-Swiss Federal Institute \\ of Technology, CH-8093 Zurich, Switzerland \\ ${ }^{3}$ Swiss Light Source, Paul Scherrer Institut, CH-5232 Villigen PSI, Switzerland \\ ${ }^{4}$ Advanced Light Source, Ernest Orlando Lawrence Berkeley National Laboratory, Berkeley, \\ California 94720, USA \\ ${ }^{5}$ Technische Universität Bergakademie Freiberg, D-09596 Freiberg, Germany
}

(Received 7 August 2009; accepted 18 September 2009; published online 30 October 2009)

\begin{abstract}
$A$-site substitution of $\mathrm{La}^{3+}$ by $\mathrm{Sr}^{2+}$ in polaron conducting $A B \mathrm{O}_{3}$-type perovskite $\mathrm{La}_{1-x} \mathrm{Sr}_{x} \mathrm{Fe}_{0.75} \mathrm{Ni}_{0.25} \mathrm{O}_{3-\delta}$ causes oxidation of $\mathrm{Fe}^{3+}$ toward $\mathrm{Fe}^{4+}$ and formation of conducting electron holes, as evidenced by $\mathrm{Fe}(2 p)$ and $\mathrm{O}(1 s)$ near edge $\mathrm{x}$-ray absorption fine structure spectra. Hole doping is reflected by linear variation of the prepeak ratio $e_{g}(\uparrow) /\left[t_{2 g}(\downarrow)+e_{g}(\downarrow)\right]$ of oxygen spectra, along with increased conductivity. The significant increase in conductivity due to $\mathrm{NiO}$ doping in $\mathrm{La}_{1-x} \mathrm{Sr}_{x} \mathrm{FeO}_{3-\delta}$ is caused by increased overlap between $\mathrm{Fe}(3 d)$ and $\mathrm{O}(2 p)$ and charge transfer from the $\mathrm{O}(2 p)$ to the $\mathrm{Ni}(3 d)$ states, as concluded from near edge $\mathrm{x}$-ray absorption fine structure spectra and ligand field multiplet calculations. (C) 2009 American Institute of Physics.
\end{abstract}

[doi:10.1063/1.3246145]

Battery and solid oxide fuel cell (SOFC) cathodes are typically based on transition metal (TM) oxides, often with noninteger average metal oxidation states. Adding large rare earth cations to the TM oxides facilitates phases with metal insulator transitions, charge ordering, superconductivity, or colossal magnetoresistance. These TM have a high oxidation state and their ground state is dominated by $\mathrm{O}(2 p)$ band holes, and not by the metal $3 d$ electrons. ${ }^{1}$

This work is about $\mathrm{La}_{1-x} \mathrm{Sr}_{x} \mathrm{Fe}_{0.75} \mathrm{Ni}_{0.25} \mathrm{O}_{3-\delta}$ (LSFN), an $A B \mathrm{O}_{3}$ perovskite. The iron in the parent compound $\mathrm{LaFeO}_{3}$ is in the high spin (HS), trivalent insulating oxidation state $\left(3 d^{5}\right)$, making it a charge transfer insulator. ${ }^{2}$

Substitution of the $A$-site $\mathrm{La}^{3+}$ by $\mathrm{Sr}^{2+}$ causes oxidation of $\mathrm{Fe}$ toward $\mathrm{Fe}^{4+}$ with $3 d^{5} \mathrm{~L}$ state, $\mathrm{L}$ denoting an electron ligand hole. ${ }^{3}$ The temperature $(T)$ dependence of the electric conductivity $\sigma(T)$ in this class of materials follows often an exponential and is known as polaron activated conductivity. ${ }^{4}$

The maximum conductivity of LaSrFe-oxide (LSF) is around $500{ }^{\circ} \mathrm{C}$, a temperature range relevant for intermediate temperature SOFC. $\mathrm{La}_{0.5} \mathrm{Sr}_{0.5} \mathrm{FeO}_{3}$ has the highest conductivity in the LSF series, but substituting $B$-site with nickel increases $\sigma(T)$ significantly to around $700 \mathrm{~S} / \mathrm{cm}^{5}$ Similar holds for $\mathrm{Sr}_{3} \mathrm{Fe} M \mathrm{O}_{7}(M=\mathrm{Fe}, \mathrm{Co}, \mathrm{Ni})$, where $\sigma(T)$ increases from $\mathrm{Fe}$ to $\mathrm{Ni}^{6}{ }^{6}$ while the $p$ - $d$ transfer integral decreases.

$\mathrm{X}$-ray spectroscopy has contributed to the understanding of hole doping by $A$-site substitution in LSF. ${ }^{7}$ In analogy to $A$-site substitution in $\mathrm{LaFeO}_{3},{ }^{8}$ substitution of $\mathrm{Sr}$ creates new features in the oxygen near edge $\mathrm{X}$-ray absorption fine structure (NEXAFS) spectra, along with increasing $\sigma(T)$.

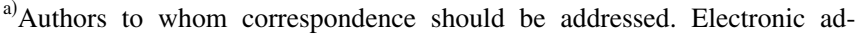
dresses: selma.erat@empa.ch and artur.braun@alumni.ethz.ch. Tel.: +41 44823 4971/4850. FAX: +41448234150.
}

We extend the previous studies toward LSFN and rationalize the quantitative correlation of conductivity and electronic structure, as obtained by $\mathrm{x}$-ray spectroscopy and supported with ligand field multiplet calculation (LFMC), in terms of $d$-type and $p$-type holes formed by $\mathrm{Sr}$ doping.

Polycrystalline LSFN with $x=0.0,0.25,0.50,0.75$, and 1.0 were prepared by solid state reaction of $\mathrm{La}_{2} \mathrm{O}_{3}$ $(>99.99 \%), \mathrm{SrCO}_{3}(99.9 \%), \mathrm{Fe}_{2} \mathrm{O}_{3}(>99.0 \%)$, and $\mathrm{NiO}$ $(99.8 \%)$ in stoichiometric proportions, calcined at $1200{ }^{\circ} \mathrm{C}$ for $4 \mathrm{~h}$ and sintered at $1400{ }^{\circ} \mathrm{C}$ for $12 \mathrm{~h}(5 \mathrm{~K} / \mathrm{min})$. The oxygen deficiency $\delta$ was obtained by thermogravimetry. $\mathrm{X}$-ray powder diffractograms (XRD) were collected with a Philips X'Pert diffractometer $(40 \mathrm{kV}, 40 \mathrm{~mA}, \mathrm{Cu} K \alpha$ radiation) at $300 \mathrm{~K}$ in steps of $0.02^{\circ}$ for $20^{\circ} \leq 2 \theta \leq 80^{\circ}$. The 4-point dc conductivity of sintered slabs (size $\sim 5 \times 3$ $\times 25 \mathrm{~mm}^{3}$ ) was measured for $300 \mathrm{~K}<T<1300 \mathrm{~K}$ in air during cooling $(2 \mathrm{~K} / \mathrm{min})$. NEXAFS spectra at $300 \mathrm{~K}$ were recorded with total electron yield at Beamline 9.3.2, Advanced Light Source, in Berkeley (photon energy range 200 $1200 \mathrm{eV}$, energy resolution up to $1 / 10000$ ). The vacuum chamber base pressure was below $5 \times 10^{-10}$ Torr. Powder samples were dispersed on conducting carbon tape and then mounted on a copper sample holder. Iron $L$-edge and oxygen spectra were recorded in steps of $0.1 \mathrm{eV}$ from 690 to $750 \mathrm{eV}$ and 520 to $560 \mathrm{eV}$, respectively.

Rietveld refinement of the XRD showed orthorhombic phase for $x=0$, and for $x=1$ cubic phases with two different cell parameters, as justified by the distinct splitting of reflections. The other samples are rhombohedral with minor amount of a tetragonal phase.

In general, LSF conductivity $\sigma(T)$ increases to a maximum upon annealing and then decreases. The effect of $T$ on $\sigma$ for LSF has been explained in terms of small-polaron hopping and follows an exponential $\sigma T \sim \exp \left(E_{p} / k T\right)$ with activation energy $E_{P}$ and Boltzmann constant $k .^{9}$ Analysis 


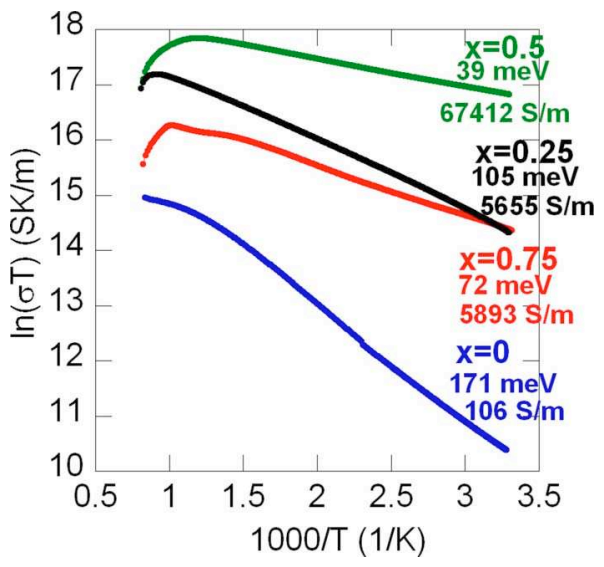

FIG. 1. (Color online) Conductivity of $\mathrm{La}_{1-x} \mathrm{Sr}_{x} \mathrm{Fe}_{0.75} \mathrm{Ni}_{0.25} \mathrm{O}_{3-\delta}$. $E_{p}$ is calculated for low temperature region $(305-465 \mathrm{~K})$.

of the Arrhenius plots for $300 \mathrm{~K}<T<1273 \mathrm{~K}$ (Fig. 1) shows that LSFN with $x=0 \quad(x=0.5)$ has the lowest (highest) conductivity and highest (lowest) $E_{P}$. The trend that LSF with $x=0.5$ have highest conductivity is thus maintained in LSFN. Upon further substitution, $\sigma(T)$ increases and $E_{P}$ decreases. The fact that LSFN has generally a higher conductivity than LSF has motivated us to study this system. LSF samples with $x=0.50$ have highest conductivity $(352 \mathrm{~S} / \mathrm{cm}$ at $823 \mathrm{~K})$. The maximum conductivity of $\mathrm{La}_{0.5} \mathrm{Sr}_{0.5} \mathrm{Fe}_{0.8} \mathrm{Ni}_{0.2} \mathrm{O}_{3}$ $\left(\mathrm{La}_{0.5} \mathrm{Sr}_{0.5} \mathrm{Fe}_{0.4} \mathrm{Ni}_{0.6} \mathrm{O}_{3}\right)$ is around $700 \mathrm{~S} / \mathrm{cm}(350 \mathrm{~S} / \mathrm{cm}){ }^{5}$ Our sample $\mathrm{La}_{0.5} \mathrm{Sr}_{0.5} \mathrm{Fe}_{0.75} \mathrm{Ni}_{0.25} \mathrm{O}_{3-\delta}$ has $678 \mathrm{~S} / \mathrm{cm}$. This maximum apparently decreases at doping levels from $20 \%$ to $60 \%$. Our sample with $x=1.0$ was very brittle and could not be subjected to conductivity measurements.

The area normalized $\mathrm{Fe} L_{2,3}$ edge spectra of LSFN in Fig. 2 show the spin-orbit split $L_{3}$ and $L_{2}$ edges of the Fe $2 p$ core hole. ${ }^{8}$ Each edge is additionally split by multiplet effects and crystal field splitting. The spectrum for $x=0$ has the overall sharpest features, i.e. a set of reasonably well separated $2 p_{3 / 2} t_{2 g}$ and $e_{g}$ resonances. Comparison with literature ${ }^{8}$ suggests and simulation by LFMC confirms-that for $x=0$ $\mathrm{Fe}$ is in $\mathrm{Fe}^{3+} 3 d^{5}$ high spin state-in similarity to $\mathrm{LaFeO}_{3}$. LFMC was made assuming octahedral symmetry with Slater
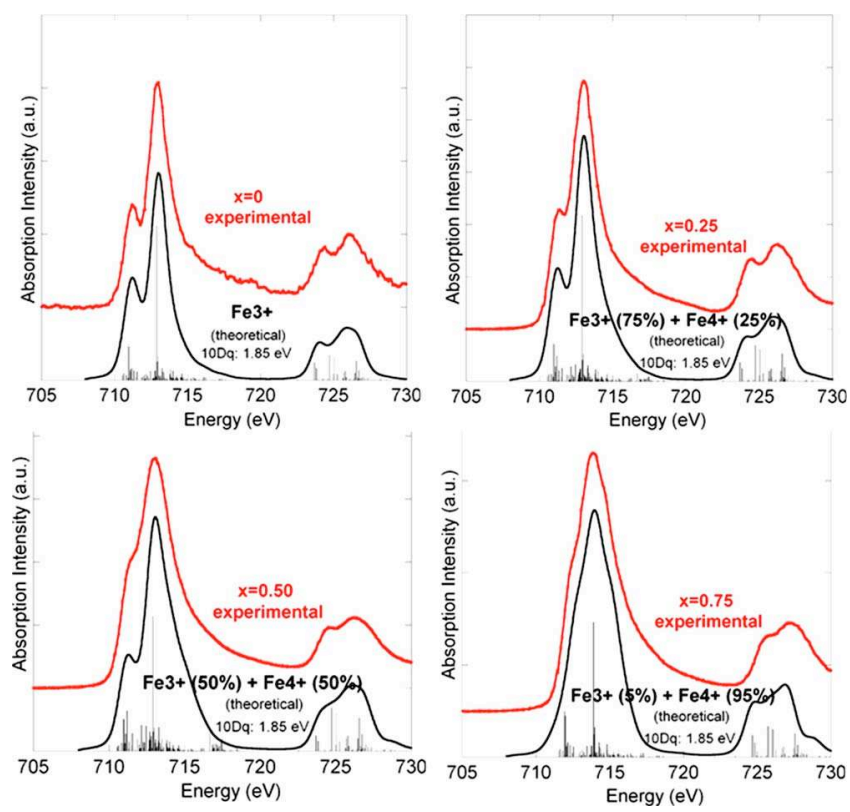

FIG. 2. (Color online) Experimental and simulated spectra of Fe $L_{2,3}$ edge.
TABLE I. Oxygen nonstoichiometry $\delta$ and valency for Fe, $\tau$ and spectral ratio $S$ in LSFN. The $\tau$ calculation is based on valency of $3+$ for Ni.

\begin{tabular}{cccccccc}
\hline \hline \multirow{2}{*}{$x$} & $\delta$ & $\begin{array}{c}\text { Ox Fe for } \\
\delta=0\end{array}$ & $\begin{array}{c}\text { Ox Fe for } \\
\delta=\exp \end{array}$ & $\begin{array}{c}\text { Ox Fe from } \\
\text { simulation }\end{array}$ & $\begin{array}{c}\tau \text { for } \\
\delta=0\end{array}$ & $\begin{array}{c}\tau \text { for } \\
\delta=\exp \end{array}$ & $S$ \\
\hline 0 & 0.000 & 3.00 & 3.00 & 3.00 & 0.00 & 0.00 & $1 / 4$ \\
$1 / 4$ & 0.035 & 3.33 & 3.24 & 3.25 & 0.33 & 0.24 & $1 / 3$ \\
$1 / 2$ & 0.053 & 3.67 & 3.53 & 3.50 & 0.67 & 0.53 & $1 / 2$ \\
$3 / 4$ & 0.069 & 4.00 & 3.82 & 3.95 & 1.00 & 0.82 & $3 / 4$ \\
\hline \hline
\end{tabular}

integrals scaled down to $50 \%$ of their atomic values in order to mimic covalency effects. With increased Sr doping, the spectra lose their discrete features. The differences in the electronic structure of $\mathrm{LaFeO}_{3}$ and $\mathrm{LaFe}_{0.75} \mathrm{Ni}_{0.25} \mathrm{O}_{3}$ are reduced Slater integrals and crystal field splitting. Our sample has stronger Slater integral reduction, indicating that the $\mathrm{Fe}$ $3 d$ orbital becomes broader, decreasing $d-d$ and $p-d$ interactions. Fe is substituted by $\mathrm{Ni}$, the $M-\mathrm{O}$ (M indicates metal) distance is reduced causing an increase in the $\mathrm{O} 2 p$ bandwidth. Thus, the $p-d$ bandgap decreases and consequently $\sigma$ increases. ${ }^{10}$ Therefore, $\mathrm{LaFe}_{0.75} \mathrm{Ni}_{0.25} \mathrm{O}_{3}$ is a semiconductor whereas $\mathrm{LaFeO}_{3}$ is an insulator at RT, despite their similar electronic configuration.

Comparison between experiment and simulation (Fig. 2) shows that LSFN with $x=0.25$ and $x=0.5$ have the percentage of $\mathrm{Fe}^{4+}$ as expected from stoichiometry. However, LSFN with $x=0.75$ shows larger amount of $\mathrm{Fe}^{4+}(95 \%)$ than suggested by stoichiometry. $\mathrm{Fe}$ is in all samples in HS with crystal field $10 D q=1.85 \mathrm{eV}$ and with Slater integral reduced to $50 \%$. We thus conclude that increasing $\mathrm{Sr}$ content changes the valency of $\mathrm{Fe}$ and $\mathrm{Ni}$, but no other electronic structure parameters. The valency of Fe, which is calculated assuming that $\mathrm{Ni}$ is $\mathrm{Ni}^{3+}$ and corrected by the experimental $\delta$, are quite close to that obtained by LFMC, except for high Sr doping level $(x=0.75)$, Table I.

The spectra in Fig. 3 represent the unoccupied O $2 p$ orbitals and reflect the empty $\mathrm{Fe} / \mathrm{Ni} 3 d$ bands at the pre-edge $(524-527 \mathrm{eV})$. The triplet at around $525 \mathrm{eV}$ is attributed to $e_{g}(\uparrow), t_{2 g}(\downarrow)$, and $e_{g}(\downarrow)$ bands. ${ }^{11}$ The intensity of the $e_{g}(\uparrow)$ peak is due to doped holes, increases upon $\mathrm{Sr}$ doping, ${ }^{8}$ and shifts slightly to the Fermi level $\left(E_{F}\right)$ depending on the hole/Sr doping concentration. Interestingly, this shift is larg-

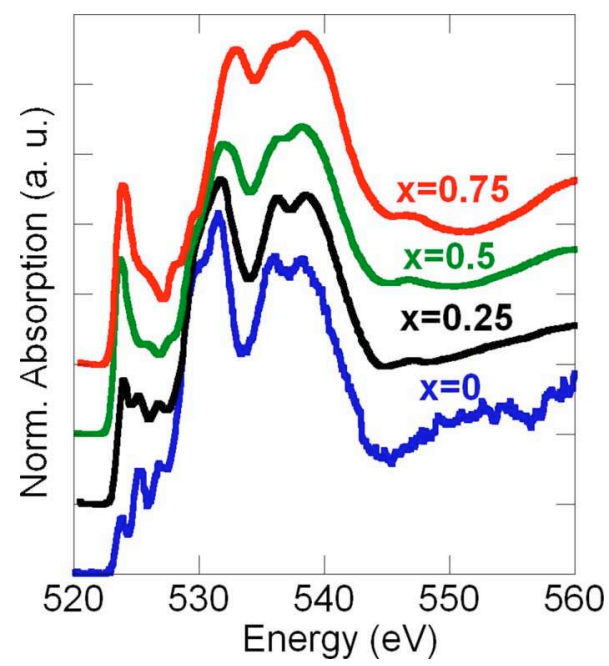

FIG. 3. (Color online) Normalized oxygen $K$ edge x-ray absorption spectra of $\mathrm{La}_{1-x} \mathrm{Sr}_{x} \mathrm{Fe}_{0.75} \mathrm{Ni}_{0.25} \mathrm{O}_{3-\delta}$. 

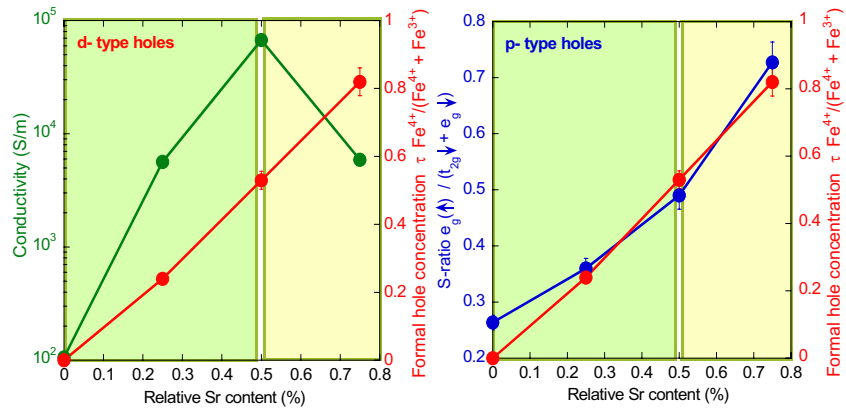

FIG. 4. (Color online) Comparison of $d$-type hole with conductivity (left), and with $S$ ratio.

est for $x=0.50$, which has maximum $\sigma$. The hole concentration $\tau$ relates to the stoichiometry as follows: ${ }^{12}$ $\mathrm{La}_{1-x} \mathrm{Sr}_{x} \mathrm{Fe}_{1-\tau}^{3+} \mathrm{Fe}_{\tau}^{4+} \mathrm{O}_{3-\delta}$. We illustrate the evolution of $\tau$ as a function of the $A$-site substitution, and begin with $x=0$ and the simplified assumption that $\delta=0, \mathrm{Ni}$ is $\mathrm{Ni}^{3+}$ and $\mathrm{La}$ and $\mathrm{Sr}$ retain their formal valences as $\mathrm{La}^{3+}$ and $\mathrm{Sr}^{2+}$. The formal charge of the iron is thus $\mathrm{Fe}^{3+}$ and $\tau=\left[\mathrm{Fe}^{4+}\right]=0$. The values corrected for the experimental $\delta$ are listed in Table I. We have recently shown ${ }^{11}$ that the ratio $S$ of spin up to spin down peaks of the upper Hubbard band in O NEXAFS spectra (pre-edge peaks) correlates with the conductivity in $A$ and $B$-site substituted LSF. We exercise this for the O spectra of our samples and find that the ratio $S$ is not for all samples close to simple rational numbers (Table I). We thus conclude that the $p$-projected electrons do not only go to $\mathrm{Fe} 3 d$ but also to Ni $3 d$ states. The evolution of $S$ which is linear in the substitution parameter $x$, and the relative Fe hole density $\tau$ are shown in Fig. 4. When $\mathrm{La}^{3+}$ is substituted by $\mathrm{Sr}^{2+}$, one hole is created. The hole concentration $\tau\left[\mathrm{Fe}^{4+} /\left(\mathrm{Fe}^{3+}\right.\right.$ $\left.+\mathrm{Fe}^{4+}\right)$ ], of LSFN increases with increasing $\mathrm{Sr}$ content, Fig. 4. $\sigma$ and $\tau$ increase upon $\mathrm{Sr}$ doping up to $50 \%$. In the high doping region, $\sigma$ decreases while $\tau$ increases. The mobility of free electrons increases with increasing $\mathrm{Sr}$ content in the low doping region: Coulomb repulsion by $\mathrm{La}^{3+}$ around oxygen in the superexchange unit $\left(\mathrm{Fe}^{4+}-\mathrm{O}^{2-}-\mathrm{Fe}^{3+}\right)$ is stronger than the barrier by $\mathrm{Sr}^{2+}$. In the high $\mathrm{Sr}$ doping region, the oxygen vacancy concentration increases along the migration pathway. ${ }^{13}$ Therefore, an electron needs higher energy to hop across $\left(\mathrm{Fe}^{3+}-\mathrm{O}^{2-}-\mathrm{Fe}^{4+}\right)$, which results in decreased mobility and consequently in a decrease of $\sigma$. This conclusion is supported by the increasing activation energy, $E_{p}=72 \mathrm{meV}$ for $x=0.75$ whereas $E_{p}=39 \mathrm{meV}$ for $x=0.50$. The interaction of $\mathrm{Fe}^{3+}-\mathrm{O}-\mathrm{Fe}^{3+}$ is antiferromagnetic whereas that of $\mathrm{Fe}^{3+}-\mathrm{O}-\mathrm{Fe}^{4+}$ is ferromagnetic. ${ }^{14}$ Once $\mathrm{Fe}^{3+}$ is oxidized to $\mathrm{Fe}^{4+}$, the number of unpaired electrons decreases and the antiferromagnetic structure is lifted in favor of ferromagnetic interaction.

We compare the $p$-type and $d$-type hole concentration depending on the $\mathrm{Sr}$ content (Fig. 4). P-type holes are created on the $\mathrm{O}$ site by transition of electrons from $\mathrm{O}(2 p)$ to $\mathrm{Fe} 3 d$ or Ni $3 d$, which scale with the ratio $S$. Since there are two different $B$-site species, $\mathrm{Fe}$ and $\mathrm{Ni}$, assessing the $p$-type hole concentration will not allow to conclude on whether electrons go to $\mathrm{Fe} 3 d$ or Ni $3 d$ orbitals. The Fe spectra basically show us Fe $d$-type hole concentration. Unfortunately, we cannot do so for the Ni because the $\mathrm{Ni}$ and
La edges coincide. We begin with $\mathrm{LaFe}_{0.75} \mathrm{Ni}_{0.25} \mathrm{O}_{3}$, which has $\mathrm{Fe}$ in $3 d^{5}$ configuration and not in $3 d^{5} \mathrm{~L}$, suggesting there is no charge transfer from $\mathrm{O}$ to $\mathrm{Fe}$. We anticipate hence no contribution from $\mathrm{Fe}$ to the $\mathrm{O} e_{g}(\uparrow)$ peak for the hole, i.e. $S=0$. However, the $S$-ratio in fact is $S=0.25$. We conclude that this charge transfer must have occurred from $\mathrm{O} 2 p$ to $\mathrm{Ni}$ $3 d$. It is known that $F e\left(\varepsilon_{d}-\varepsilon_{p}\right)>N i\left(\varepsilon_{d}-\varepsilon_{p}\right)$, where $\varepsilon_{d}$ and $\varepsilon_{p}$ are the energy of $3 d$ and $2 p$ states of $\mathrm{Fe} / \mathrm{Ni}$ and oxygen, respectively. ${ }^{15}$ As is shown in Fig. 4 (right), increasing the $\mathrm{Sr}$ content increases the $\mathrm{Fe}$ oxidation state and the $S$-ratio. When the $p$-electrons go to $\mathrm{Fe} 3 d$ orbitals, $\mathrm{Fe}$ should become reduced. Therefore, we can also conclude for the Sr doped samples that the charge transfer occurs predominantly between oxygen $2 p$ and Ni $3 d$. In LSFN we showed that increasing the $\mathrm{Sr}$ content increases the number of $d$-type $\left[\mathrm{Fe}^{4+} /\left(\mathrm{Fe}^{4+}+\mathrm{Fe}^{3+}\right)\right]$ and $p$-type holes $\left\{\left[e_{g}(\uparrow) / t_{2 g}(\downarrow)\right.\right.$ $\left.+e_{g}(\downarrow)\right\} .{ }^{11}$ We conclude that charge transfer occurred mostly from $\mathrm{O}$ to $\mathrm{Ni}$, and not to $\mathrm{Fe}$. The conductivity which has contributions from $d$ - and $p$-holes increases up to $50 \%$ of $\mathrm{Sr}$ doping. At low $\mathrm{Sr}$ doping level, we find that the $\mathrm{Fe}^{4+}$ concentration determined by LFMC is similar to the $\mathrm{Sr}$ concentration. At high $\mathrm{Sr}$ doping, the conductivity decreases while the $d$ - and the $p$-type hole concentration increase, and this because of the vacancies created along the migration pathway. Increasing the $\mathrm{Sr}$ content increases the $\mathrm{Fe}^{4+}$ concentration, but does not affect crystal field $(10 D q$ $=1.85 \mathrm{eV}$ ) and the Fe spin state.

Funding by E.U. MIRG Grant No. CT-2006-042095 and Swiss NSF Grant No. 200021-116688. The ALS is supported by the Director, Office of Science/BES, of the U.S. DoE, Grant No. DE-AC02-05CH11231.

${ }^{1}$ J. Zaanen, G. A. Sawatzky, and J. W. Allen, Phys. Rev. Lett. 55, 418 (1985).

${ }^{2}$ G. R. Hearne, M. P. Pasternak, R. D. Taylor, and P. Lacorre, Phys. Rev. B 51, 11495 (1995)

${ }^{3}$ H. Wadati, D. Kobayashi, H. Kumigashira, K. Okazaki, T. Mizokawa, A. Fujimori, K. Horiba, M. Oshima, N. Hamada, M. Lippmaa, M. Kawasaki, and H. Koinuma, Phys. Rev. B 71, 035108 (2005).

${ }^{4}$ T. Montini, M. Bevilacqua, E. Fonda, M. F. Casula, S. Lee, C. Tavagnacco, R. J. Gorte, and P. Fornasiero, Chem. Mater. 21, 1768 (2009).

${ }^{5}$ R. Chiba, F. Yoshimura, and Y. Sakurai, Solid State Ionics 152, 575 (2002).

${ }^{6}$ M. Abbate, H. Ascolani, L. Mogni, F. Prado, and A. Caneiro, Physica B 354, 7 (2004)

${ }^{7}$ A. Braun, J. Richter, A. S. Harvey, S. Erat, A. Infortuna, A. Frei, E. Pomjakushina, B. S. Mun, P. Holtappels, U. Vogt, K. Conder, L. J. Gauckler, and T. Graule, Appl. Phys. Lett. 93, 262103 (2008).

${ }^{8}$ M. Abbate, F. M. F. de Groot, J. C. Fuggle, A. Fujimori, O. Strebel, F. Lopez, M. Domke, G. Kaindl, G. A. Sawatzky, M. Takano, Y. Takeda, H. Eisaki, and S. Uchida, Phys. Rev. B 46, 4511 (1992).

${ }^{9}$ W. H. Jung, Physica B 299, 120 (2001).

${ }^{10}$ L. Mogni, F. Prado, H. Ascolani, M. Abbate, M. S. Moreno, A. Manthiram, and A. Canerio, J. Solid State Chem. 178, 1559 (2005).

${ }^{11}$ A. Braun, D. Bayraktar, S. Erat, A. S. Harvey, D. Beckel, J. A. Purton, P. Holtappels, L. J. Gauckler, and T. Graule, Appl. Phys. Lett. 94, 202102 (2009).

${ }^{12}$ J. C. Grenier, N. Ea, and M. Pouchard, Mater. Res. Bull. 19, 1301 (1984).

${ }^{13}$ M. V. Patrakeev, I. A. Leonidov, V. L. Kozhevnikov, and K. R. Poeppelmeier, J. Solid State Chem. 178, 921 (2005).

${ }^{14}$ K. S. Ryu, S. J. Lee, and C. H. Yo, Bull. Korean Chem. Soc. 15, 256 (1994).

${ }^{15}$ P. Mahadevan, N. Shanthi, and D. D. Sarma, Phys. Rev. B 54, 11199 (1996). 